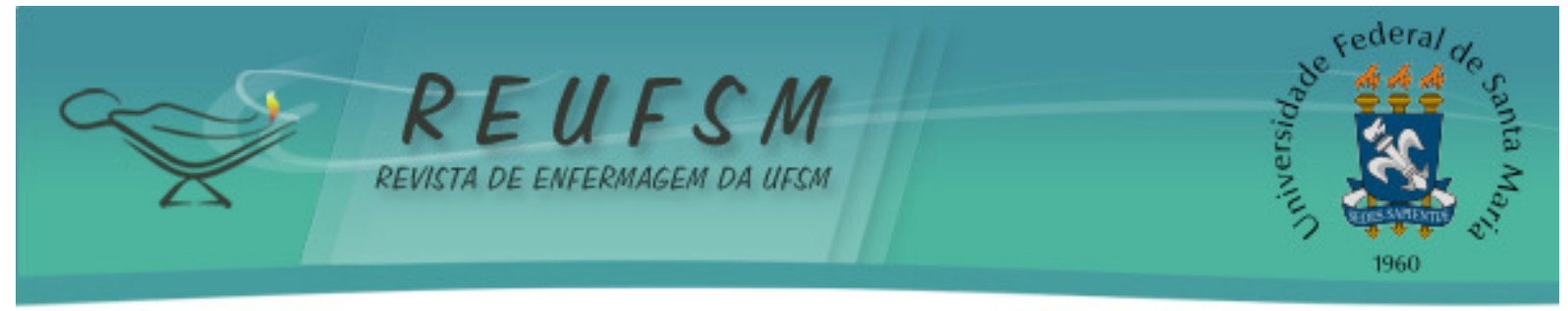

ARTIGO ORIGINAL

\title{
CONHECIMENTO DO ENFERMEIRO SOBRE INSUFICIÊNCIA CARDÍACA EM HOSPITAL GERAL
}

NURSES' KNOWLEDGE ON HEART FAILURE IN GENERAL HOSPITAL

CONOCIMIENTO DEL ENFERMERO SOBRE INSUFICIENCIA CARDIACA EN HOSPITAL GENERAL

\author{
Cátia Giovana Dias Machado ${ }^{1}$ \\ Gilciane Bolzan Wansing ${ }^{2}$ \\ Cristini Klein ${ }^{3}$ \\ Maria Antonieta Pereira de Moraes ${ }^{4}$ \\ Eneida Rejane Rabelo-Silva ${ }^{5}$
}

Doi: $10.5902 / 2179769211633$

RESUMO: Objetivos: verificar entre os enfermeiros de dois hospitais gerais o conhecimento da síndrome de Insuficiência Cardíaca (IC) e os fatores que interferem nas orientações prestadas aos pacientes. Métodos: o conhecimento foi avaliado pelo Questionário de conhecimento para enfermeiros sobre IC (Q-CENIC), composto por 14 questões. Também foram realizados questionamentos relacionados à orientação, limitações para orientação, e auto avaliação sobre conhecimentos da IC. Resultados: foram avaliados 51 enfermeiros, destes $82,4 \%$ atingiram o percentual de $70 \%$ de acertos. Sintomas de IC avançada e uso de sildenafil foram questões que tiveram percentuais menores de acertos. Mais de $70 \%(n=40)$ dos enfermeiros orienta os pacientes sobre IC e autocuidado. $\mathrm{O}$ fator que mais interfere nas orientações é a falta de tempo. Conclusão: os resultados indicam que os enfermeiros orientam e apresentaram conhecimento satisfatório sobre o manejo da síndrome de IC. A falta de tempo foi a principal dificuldade para otimização desse processo.

Descritores: Insuficiência cardíaca; Conhecimento; Enfermagem.

ABSTRACT: Objective: to evaluate the knowledge of nurses from two general hospitals on heart failure (HF) syndrome and the factors that interfere with the guidance they provide to patients. Methods: knowledge was assessed by the 14-item Nurses' Knowledge of Heart Failure (NKHF) questionnaire. Participants were also asked about guidance, guidance limitations, and self-assessment of knowledge on HF. Results: fifty-one nurses were evaluated, $82.4 \%$ of which achieved a percentage of $70 \%$ of correct answers. Symptoms of advanced HF and use of sildenafil were the items that had the lowest

\footnotetext{
${ }^{1}$ Enfermeira. Especialista em Cardiologia. Enfermeira Assistencial do Hospital de Beneficência Cachoeira do Sul, Cachoeira do Sul, Rio Grande do Sul, Brasil. catiagiovanamachado@bol.com.br

${ }^{2}$ Enfermeira. Especialista em Cardiologia. Enfermeira Assistencial do Hospital Santa Cruz, Santa Cruz do Sul, Rio Grande do Sul, Brasil. gilcianew@unisc.br

${ }^{3}$ Enfermeira. Doutoranda do Programa de Pós Graduação em Ciência Médicas da Universidade Federal do Rio Grande do Sul (UFRGS). Enfermeira Assistencial do Serviço de Enfermagem em Terapia Intensiva do Hospital de Clinicas de Porto Alegre, Rio Grande do Sul, Brasil. ckklein@hcpa.ufrgs.br

${ }^{4}$ Enfermeira. Doutora em Ciências da Saúde: Cardiologia. Professora Pós-Graduação Lato-Sensu: Enfermagem em Cardiologia do Instituto de Cardiologia do RGS (IC-FUC). Coordenadora Programa de Residência Multidisciplinar Integrada em Saúde: Enfermagem em Cardiologia do IC-FUC. Líder do Grupo de Estudo e Pesquisa sobre Práticas de Cuidado na Promoção da Saúde do Adulto com Alterações Cardiovasculares PROCARDIO. Porto Alegre, Rio Grande do Sul, Brasil.antonieta_moraes@uol.com.br

${ }^{5}$ Enfermeira. Doutora em Ciências Biológicas: Fisiologia. Professor Associado da Escola de Enfermagem da Universidade Federal do Rio Grande do Sul. Coordenadora da Clínica de Insuficiência Cardíaca do Hospital de Clinicas de Porto Alegre. Líder do Grupo de Pesquisa no Cuidado ao Adulto e Idoso - GEPECADI. Porto Alegre, Rio Grande do Sul, Brasil.eneidarabelo@gmail.com
} 


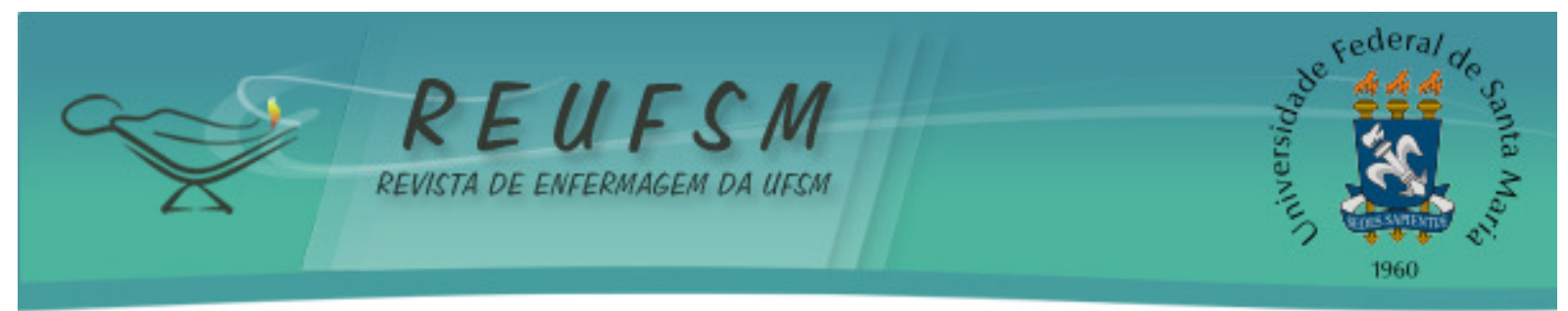

percentages of correct answers. More than $70 \%(n=40)$ of nurses provided patients with guidance on HF and self-care. The factor that most interfered with guidance was lack of time. Conclusion: results indicate that nurses advise patients and have satisfactory knowledge on the management of HF syndrome. Lack of time was the main difficulty for optimizing this process.

Descriptors: Heart failure; Knowledge; Nursing.

RESUMEN: Objetivos: verificar el conocimiento sobre insuficiencia cardiaca (IC) entre enfermeros de dos hospitales generales y los factores que interfieren en las orientaciones a los pacientes. Métodos: se evaluó el conocimiento mediante el Cuestionario de Conocimiento sobre IC para enfermeros (Q-CENIC), compuesto de 14 preguntas. También se cuestionó sobre la orientación, limitaciones a la orientación, y autoevaluación de conocimientos sobre IC. Resultados: se evaluaron 51 enfermeros, el 82,4\% de los cuales llegaron al 70\% de aciertos. Síntomas de IC avanzada y uso de sildenafil fueron los tópicos con los menores porcentajes de acierto. Más del $70 \%(n=40)$ de los enfermeros orienta a los pacientes sobre IC y autocuidado. El factor que más interfiere en las orientaciones es falta de tiempo. Conclusión: los resultados indican que los enfermeros orientan y presentan conocimiento satisfactorio sobre el manejo de la IC. La falta de tiempo fue la principal dificultad para la optimización del proceso.

Descriptores: Insuficiencia cardíaca; Conocimiento; Enfermería.

\section{INTRODUÇÃO}

A insuficiência cardíaca (IC) é reconhecida como um crescente problema de saúde pública. Relacionadas a esta síndrome estão elevadas taxas de morbidade e mortalidade, que contribuem para o aumento nas hospitalizações e re-hospitalizações. ${ }^{1}$

Estudos realizados ao longo das últimas décadas identificaram que a falta de adesão ao tratamento, devido ao desconhecimento do paciente sobre o mesmo, é uma das principais causas de descompensações e internações hospitalares associadas à $\mathrm{IC} \cdot{ }^{1-5}$ Neste cenário, a equipe multidisciplinar, coordenada por enfermeiros, tem desenvolvido e implementado estratégias de educação e acompanhamento sobre a doença e o autocuidado. ${ }^{6}$

Estudo recente demonstrou que pacientes tem benefício no autocuidado e entendimento da doença, quando as orientações são fornecidas de modo repetitivo, durante a internação por descompensação da IC. ${ }^{7}$ Neste contexto, o enfermeiro necessita ter ciência sobre as necessidades de informações a serem transmitidas ao paciente com IC. Deste modo, devido à importância da temática, nos últimos anos são crescentes os trabalhos verificando conhecimento dos enfermeiros sobre esta síndrome de alta mortalidade.

0 primeiro estudo que avaliou este tema incluiu 300 profissionais, em cinco instituições hospitalares dos Estados Unidos. Este estudo mostrou que os enfermeiros apresentavam conhecimento insuficiente sobre o manejo da IC, considerando um percentual satisfatório de $87,5 \%$ de acertos no instrumento Nurse's Knowledge of Heart Failure (NKHF). ${ }^{8}$ Resultados semelhantes utilizando o mesmo instrumento foram demonstrados na avaliação de 51 enfermeiros hospitalares norte americanos. ${ }^{9}$

Considerando a importância do conhecimento do enfermeiro no manejo clínico de pacientes com IC, e a indisponibilidade de instrumentos para este fim no país, nosso grupo de pesquisa realizou a tradução, adaptação e validação do questionário NKHF para uso no Brasil. Após este processo, o questionário foi denominado Questionário de Conhecimento de Enfermeiros sobre IC (Q-CENIC). ${ }^{10}$ 


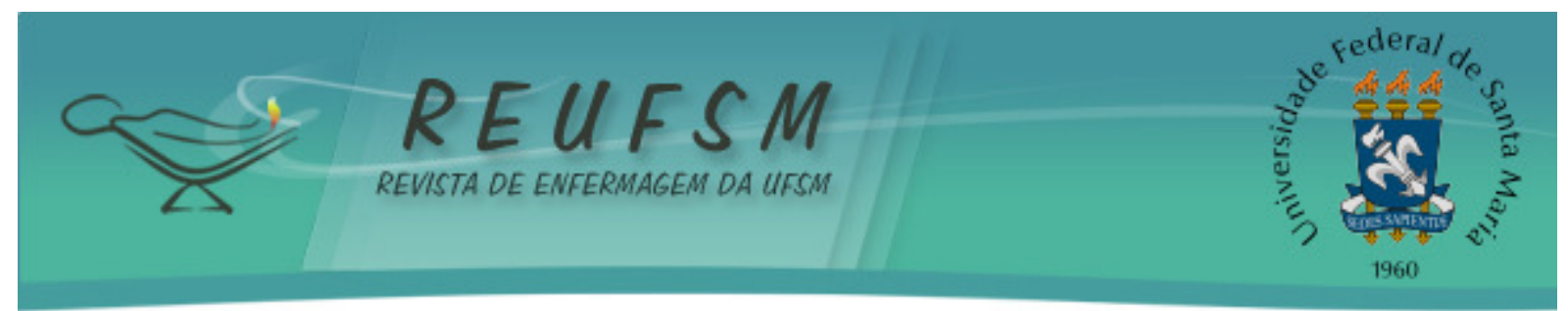

Diante da disponibilidade de um questionário validado para uso no Brasil, e a necessidade de qualificar a assistência ao paciente com IC, vimos a oportunidade de avaliar o conhecimento dos enfermeiros sobre o manejo clínico da IC e suas dificuldades frente a este processo. Deste modo, buscou-se verificar entre os enfermeiros de dois hospitais gerais o conhecimento da síndrome de IC e os fatores que interferem nas orientações prestadas aos pacientes.

\section{MÉTODO}

Estudo transversal, desenvolvido de janeiro a abril de 2011. A coleta de dados foi realizada, simultaneamente em dois hospitais gerais, públicos, de médio porte, localizados no interior do Estado do Rio Grande do Sul.

Foram incluídos todos os enfermeiros que trabalham nas instituições em estudo há quatro meses ou mais, prestam cuidados a pacientes adultos nas unidades clínica e cirúrgica, emergência, unidade de terapia intensiva. Optou-se por um instrumento de coleta de dados composto por duas partes: a primeira constituída por questões referentes as características gerais da amostra, perguntas relacionadas a orientação, limitações para orientação e auto avaliação sobre conhecimentos da IC, e a segunda constituída pelo instrumento Q-CENIC.

O Q-CENIC é utilizado para avaliação do conhecimento dos enfermeiros sobre IC e seu manejo clínico. Foi adaptado do instrumento americano $\mathrm{NKHF}^{8}$, e validado para uso no Brasil. ${ }^{10}$ O Q-CENIC compreende 14 questões de verdadeiro ou falso sobre dieta, líquidos e peso, sinais e sintomas, tratamento, atividade física e sexual. Os autores do instrumento NKHF preconizaram como tendo conhecimento suficiente sobre o manejo clínico da IC o enfermeiro que tem um percentual de acerto $\geq 87,5 \%$ das questões corretas do questionário. ${ }^{10}$ Não foram relatados estudos sobre características psicométricas referentes aos pontos de corte dos instrumentos NKHF e o Q-CENIC, para discriminar os enfermeiros com e sem conhecimento suficiente sobre o manejo da IC. Deste modo, em nosso estudo, por ser realizado em dois hospitais gerais, consideramos como tendo conhecimento suficiente sobre o manejo clínico da IC o enfermeiro que atingisse $\geq 70 \%$ das questões do questionário.

O projeto de pesquisa foi aprovado pelo Comitê de Ética e Pesquisa das instituições, sob os números $2721 / 10$ e $4520 / 10$. O estudo seguiu todas as recomendações constantes na Resolução 196/96 do Conselho Nacional de Saúde. ${ }^{11}$ Todos os enfermeiros foram incluídos, após assinatura do Termo de Consentimento Livre e Esclarecido.

\section{Análise estatística}

Os dados foram inseridos em uma tabela do Programa Excel for Windows. As análises foram realizadas utilizando o pacote estatístico Statistical Package for Social Sciences, versão 18.0. As variáveis contínuas foram expressas como média \pm desvio padrão ou mediana e intervalo interquartil. As variáveis categóricas foram expressas com frequências relativas e percentual.

\section{RESULTADOS E DISCUSSÃO}

Neste estudo buscou-se investigar o conhecimento e o manejo clínico dos enfermeiros em pacientes hospitalizados acometidos por IC utilizando o questionário QCENIC. ${ }^{10}$ Foram incluídos no estudo 51 enfermeiros. A idade média dos enfermeiros foi de $32 \pm 6$ anos, predominando o sexo feminino. Metade da amostra composta por profissionais com até cinco anos de formado, com especialização completa e atuando em unidades de 


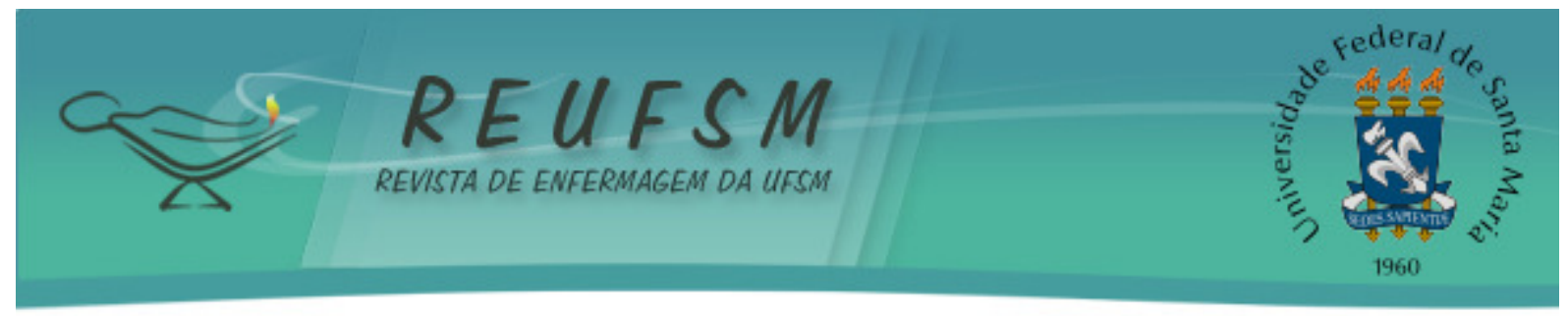

emergência, unidade de terapia intensiva, hemodinâmica seguido das unidades de internação clínica e cirúrgica, Tabela 1.

Tabela 1 - Características gerais da amostra de enfermeiros. Santa Cruz, 2011.

\begin{tabular}{lc}
\hline Características & $\mathbf{N}=\mathbf{5 1}$ \\
Idade, anos & $32 \pm 6$ \\
Sexo (feminino) & $38(76)$ \\
Especialização completa & $26(51)$ \\
Graduação & $12(23,5)$ \\
Especialização curso & $7(13,7)$ \\
Mestrado completo/curso & $5(9,8)$ \\
Tempo de formação superior* & $5(4-9)$ \\
Tempo de trabalho na instituição* & $2(1,4-5,7)$ \\
Unidade de Emergência/ UTI / Hemodinâmica & $28(54,9)$ \\
Unidade internação clínica/cirúrgica & $26(51)$ \\
Unidade de hemodiálise / Oncologia & $5(9,8)$ \\
\hline N (\%); valores expressos como média \pm desvio padrão; descritos como mediana e intervalo \\
interquartil. * em anos
\end{tabular}

No desempenho geral no teste, $82,4 \%$ dos enfermeiros avaliados atingiram o percentual preconizado pelas autoras do presente estudo de $70 \%$ para que o conhecimento sobre IC e autocuidado fosse considerado satisfatório. No estudo dos autores que desenvolveram o questionário, estes consideraram conhecimento satisfatório no teste, o enfermeiro cujo percentual de acertos foi maior que $87,5 \%$ das questões. ${ }^{8}$ Da mesma forma, no estudo que realizou a validação do questionário para uso no Brasil, aplicado em dois cenários (hospital geral e especializado em cardiologia) somente $20 \%$ dos enfermeiros atingiram este percentual. ${ }^{10}$ Igualmente um estudo norte americano recente, utilizando a mesma metodologia, verificou que somente $8,9 \%$ dos enfermeiros atingiu $85 \%$ ou mais de acertos em questões do teste. ${ }^{12}$ Visto que o ponto de corte do presente estudo é diferente dos demais, as comparações com estes têm limitações. Do mesmo modo, o presente estudo foi aplicado em uma amostra específica de enfermeiros do interior do Rio Grande do Sul, e provavelmente não representa o conhecimento dos enfermeiros de todo estado e país. Assim, sugere-se aplicar este questionário em outros centros para verificar as necessidades de treinamento sobre manejo da IC em outros cenários brasileiros.

A Tabela 2 ilustra o conhecimento dos enfermeiros no manejo clínico de pacientes com IC. É possível observar que para a maioria das questões os acertos foram superiores a $80 \%$ (64\%). 


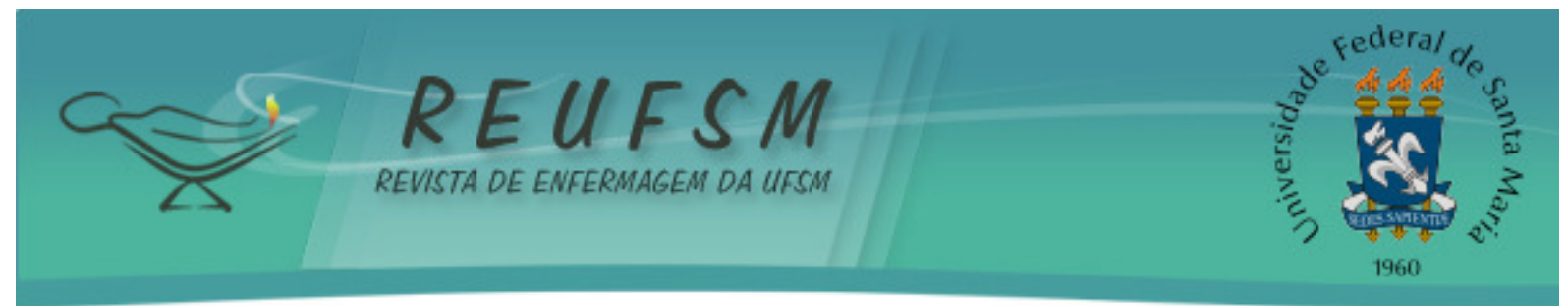

Tabela 2 - Conhecimento dos enfermeiros do manejo clínico dos pacientes com insuficiência cardíaca, Santa Cruz, 2011.

\section{Questões de verdadeiro e falso}

Pacientes estáveis podem manter atividade sexual (V)

Classe de IC e idade $x$ orientação sobre atividade física (V)

Acréscimo de $1400 \mathrm{Kg}$ em $48 \mathrm{H}$, deve ser considerado $(\mathrm{F})$

Sinais de congestão desaparecem, ñ precisa mais cuidados $(F)$

Todos os pacientes podem tomar líquidos à vontade $(\mathrm{F})$

Dispnéia ao acordar, sentar na cama melhora, é retenção (V)

Tratamento adequado cura IC (F)

Todos os pacientes devem restringir sal na sua dieta (V)

Evitar carnes defumadas e enlatados (V)

Abdômem globoso indica retenção de líquidos (V)

Dormir com mais de 1 travesseiro, não significa piora da IC (F)

Controle peso deve ser comparado com o dia anterior (V)

Sildenafil 24 horas após a suspensão do nitrato (V)

Tosse, náuseas e diminuição do apetite, IC avançada (V)
Questionário

Acertos (\%)

$\mathrm{n}=\mathbf{5 1}$

$51(100)$

$51(100)$

$47(92,2)$

$46(90,2)$

$45(88,2)$

$44(86,3)$

$44(86,3)$

$43(84,3)$

$43(84,3)$

$37(72,5)$

$33(64,7)$

$31(60,8)$

$27(52,9)$

$25(49)$

Variáveis categóricas expressas com $\mathrm{n}(\%)$.

Questões sobre sinais e sintomas de IC avançada, o controle diário do peso e uso de sildenafil foram as que tiveram percentuais menores de acertos. Estes achados são diferentes dos resultados dos estudos norte americanos que demonstraram que os enfermeiros têm entre 98 e $100 \%$ de acertos nestas questões. ${ }^{8-9}$ A abordagem multidisciplinar na IC, assim como o cuidado por equipe especializada podem ter contribuído ou melhorado esses achados nos estudos norte americanos.

Entretanto, no presente estudo, questões relativas à restrição hidrossalina, cuidados frente a sinais de descompensação e atividade física, foram acertadas pela maioria dos enfermeiros, diferente dos estudos prévios que aplicaram o mesmo questionário. Salienta-se que de fato estas questões são relevantes e parte essencial do manejo complexo que envolve pacientes com IC. ${ }^{13}$

Considerando as orientações prestadas pelo enfermeiro ao paciente com IC, verificamos que mais de $70 \%$ dos enfermeiros afirmam que orientam os pacientes sobre a síndrome e autocuidado. Com relação aos pacientes que recebem as orientações, aqueles que estão no ambiente hospitalar são mais propícios a receberam as informações, principalmente por estarem mais sensibilizados com o impacto causado pela internação e pelos sintomas de descompensação. ${ }^{6,14}$

A maioria dos enfermeiros orienta os pacientes sobre IC e autocuidado (78\%), e destes, $45 \%$ orientam todos os pacientes, conforme os itens abordados no Q-CENIC. Dentre os fatores que mais interferem nas orientações, a falta de tempo para orientar foi o mais citado (85\%), seguido de outros fatores como momento inadequado para orientação, ausência da descrição do diagnóstico no prontuário e fatores ambientais. Estes resultados estão demonstrados na Tabela 3. 


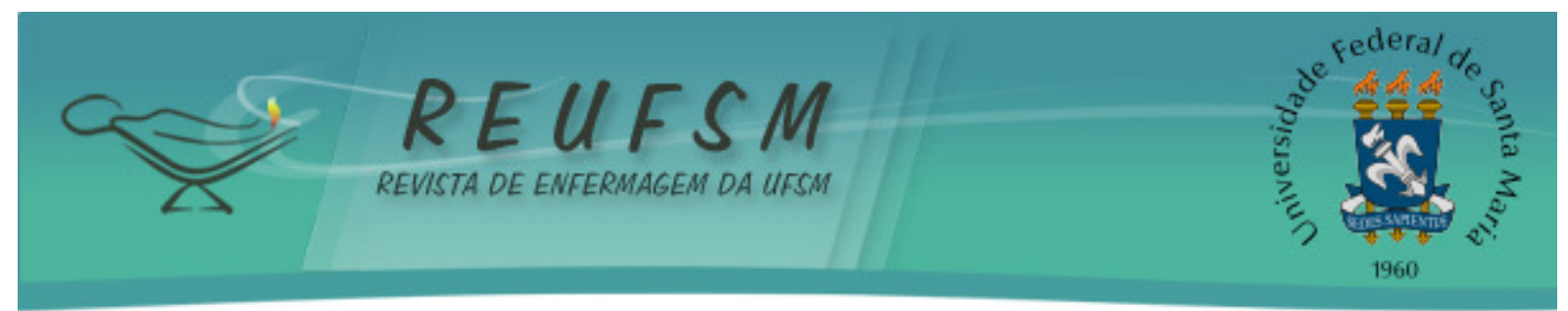

Tabela 3 - Fatores que interferem nas orientações sobre IC e autocuidado para todos os pacientes, Santa Cruz, 2011.

\begin{tabular}{lc}
\hline Fatores que interferem nas orientações & $\mathbf{N}(\mathbf{\%})$ \\
& $(\mathbf{n}=\mathbf{1 8})$ \\
\hline Falta de tempo & $20(87)$ \\
Fatores ambientais & $9(37,5)$ \\
Falta de descrição do diagnóstico & $8(34,8)$ \\
Momento inadequado & $8(34,8)$ \\
Falta de conhecimento & $4(17,4)$ \\
\hline
\end{tabular}

Dos enfermeiros, $50 \%$ classificaram seu conhecimento como bom em relação à IC, seguido por $43 \% \quad(n=22)$ que consideravam ser regular. Em sua maioria, $78 \%$ afirmam orientar os pacientes com IC sobre auto cuidado. Destes, $85 \%$ priorizam orientar pacientes que reinternam por IC, e $60 \%$ orientam os pacientes graves. Mais de $50 \%$ dos enfermeiros fazem a orientação para pacientes e familiares que demonstram mais entendimento das questões relacionadas à doença e também para aqueles onde a equipe médica descreve o diagnóstico da doença no prontuário do paciente.

Considerando o conhecimento sobre IC como a base para o profissional que irá prestar orientações ao paciente, é imprescindível que o enfermeiro tenha conhecimento suficiente sobre a síndrome para poder orientar o paciente adequadamente. A literatura já demonstra benefícios quanto às intervenções de educação. Em uma revisão sistemática, com 74 estudos, a abordagem multidisciplinar para pacientes com IC não apenas reduz internação, como também é um método eficaz para diminuir a mortalidade. ${ }^{15}$ Outro estudo, realizado em um hospital comunitário na Alemanha, verificou que $42 \%$ das internações dos pacientes com IC estão relacionadas à não-adesão ao regime medicamentoso, sabendo que um dos fatores para este fato são as orientações fornecidas e a forma com que são transmitidas. ${ }^{16}$ Corroborando com esses resultados, uma coorte com 501 pacientes, mostrou associação entre adesão e conhecimento, tendo como resultados menos crises de descompensação e readmissões. ${ }^{17}$ Neste mesmo estudo, o nível educacional foi relacionado com a adesão ao tratamento. ${ }^{17} \mathrm{Em}$ um ensaio clinico randomizado brasileiro, com uma população de 120 pacientes, grupo intervenção com contato telefônico e grupo controle sem contato telefônico, os resultados demonstraram que a intervenção educativa de enfermagem realizada durante o período de hospitalização trouxe melhora no conhecimento sobre a IC e autocuidado dos pacientes, independente do contato telefônico após a alta hospitalar. ${ }^{7}$

Orientações para o autocuidado são atividades desafiadoras para o paciente, seus familiares e equipe de saúde. A abordagem multidisciplinar tem sido a melhor estratégia, desde a avaliação, até o acompanhamento do que foi implementado. ${ }^{18} \mathrm{Um}$ estudo afirma que as orientações precisam ser repetitivas e de modo muito encorajador, pois os membros da equipe multidisciplinar possuem papéis importantes na educação de pacientes com IC. ${ }^{14}$

Dois estudos clínicos randomizados, um brasileiro e um colombiano, com pacientes com IC descompensada, que receberam visitas domiciliares mais a tele-enfermagem, trazem resultados benéficos e significativos com relação ao conhecimento e auto cuidado desses pacientes. ${ }^{19-20}$ Isso vem ao encontro de que o preparo dos enfermeiros com relação ao conhecimento e o esforço da equipe com a colaboração do paciente e de seus cuidadores poderão trazer benefícios no entendimento da doença e autocuidado resultando em melhores desfechos clínicos. ${ }^{21}$

Cabe ressaltar que o presente estudo tem limitações, visto que o ponto de corte é diferente dos estudos anteriormente descritos utilizando o Q-CENIC e o NKHF. Porém, enfatizamos que os enfermeiros lotados nas duas instituições em estudo apresentaram resultados satisfatórios $(82,4 \%)$, sobre o manejo clínico de pacientes com IC, considerando 


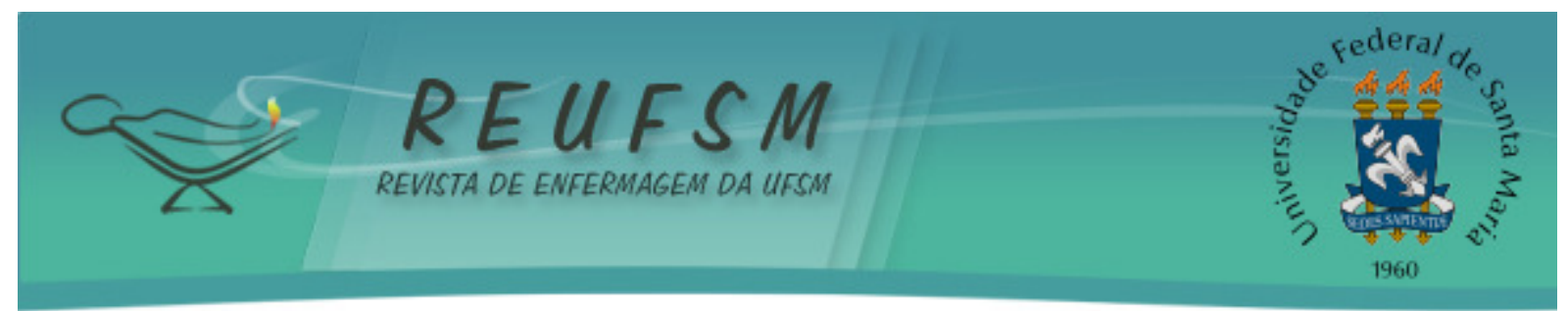

como suficiente $70 \%$ de acertos. Deste modo, apesar do escore satisfatório de conhecimento dos enfermeiros desse estudo, sugere-se trabalhos de educação permanente, com implementação de seminários de atualização e grupos de estudo, visando acompanhar a dinâmica de atualização sobre esta síndrome tão prevalente.

\section{CONCLUSÕES}

Resultados desse estudo indicam que a maioria dos enfermeiros tem conhecimento satisfatório sobre aspectos importantes que necessitam ser abordados com pacientes com IC. Os enfermeiros orientam sobre a doença e o autocuidado, sendo à falta de tempo a principal dificuldade para otimização desse processo. Profissionais de hemodiálise e oncologia demonstraram um maior percentual de conhecimento em âmbito geral. Os aspectos relacionados aos sinais e sintomas de IC avançada e uso de sildenafil foram às questões menos conhecidas.

\section{REFERÊNCIAS}

1. Barretto ACP, Del Carlo CH, Cardoso JN, Morgado PC, Munhoz RT, Eid MO, et al. ReHospitalizações e Morte por Insuficiência Cardíaca- Índices Ainda Alarmantes. Arq Bras Cardiol. 2008;91(5):335-41.

2. Bennett SJ, Huster GA, Baker SL, Milgrom LB, Kirchgassner A, Birt J, et al. Characterization of the precipitants of hospitalization for heart failure decompensation. Am J Crit Care. 1998; 7(3): 168-74.

3. Opasich C, Febo O, Riccardi PG, Traversi E, Forni G, Pinna G, et al. Concomitant factors of decompensation in chronic heart failure. Am J Card. 1996; 78(3): 354-7.

4. Van Der Wal MHL, Jaarsma T. Adherence in heart failure in the elderly: problem and possible solutions. Int J Cardiol. 2008; 125(2): 203-8.

5. Tsuyuki RT, McKelvie RS, Arnold JM, Avezum A Jr, Barretto AC, Carvalho AC, et al. Acute precipitants of congestive heart failure exacerbations. Arch Intern Med. 2001; 161(19):2337-42.

6. Aliti GB, Rabelo ER, Domingues FB, Clausell N. Educational Settings in the Management of Patients with Heart Failure. Rev Lat Am Enfermagem. 2007; 15(2): 344-9.

7. Domingues FB, Clausell N, Aliti GB, Dominguez DR, Rabelo ER. Education and telephone monitoring by nurses of patients with heart failure: randomized clinical trial. Arq Bras Cardiol. 2011; 96 (3): 233-9.

8. Albert NM, Collier S, Sumondi V, Wilkinson S, Hammel JP, Vopat L, et al. Nurse's Knowledge of Heart Failure Education Principles. Heart Lung. 2002; 31 (2): 102-12.

9. Washburn SC, Hornberger CA, Klutman A, Skinner L. Nurse's Knowledge of Heart Failure Education Topics as Report in a Small Midwestern Community Hospital. J Cardiovasc Nurs. 2005; 20 (3): 215-20.

10. Klein C, Linch GF, de Souza EN, Mantovani VM, Goldmeier S, Rabelo ER. Cross- cultural adaptation and validation of a questionnaire on what nurses know of heart failure. Rev Gaucha Enferm. 2012;33(1):19-25. 


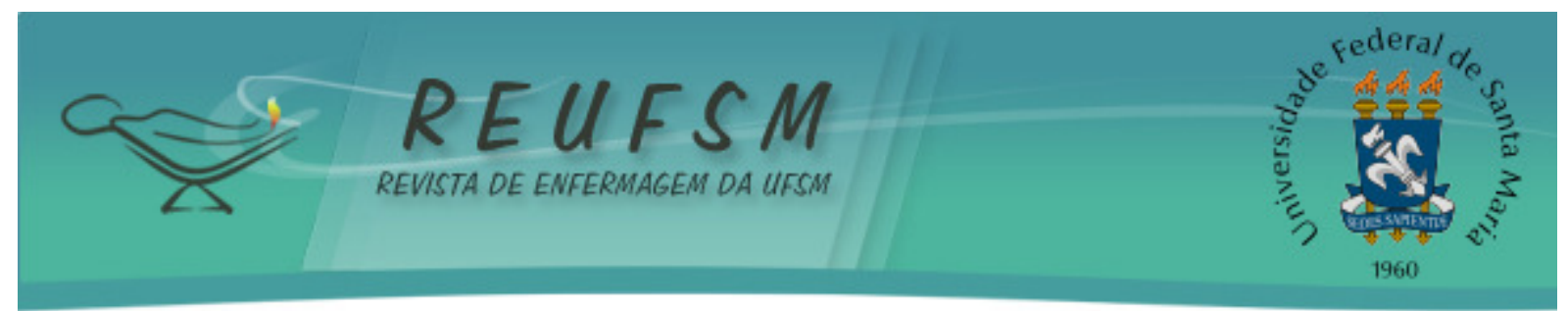

11. Conselho Nacional de Saúde (BR). Resolução n. 196/96: dispõe sobre pesquisa envolvendo seres humanos. Brasília (DF) [acesso em 2010 dez 1]. Disponível em: http://www.ufrgs.br/bioetica/res19696.htm.

12. Mahramus TL, Penoyer DA, Sole ML, Wilson D, Chamberlain L, Warrington W. Clinical nurse specialist assessment of nurses' knowledge of heart failure. Clin Nurse Spec. 2013;27(4):198-204.

13. Bocchi EA, Braga FG, Ferreira SM, Rohde LE, Oliveira WA, Almeida DR et al. III Diretriz Brasileira de Insuficiência Cardíaca Crônica. Arq Bras Cardiol. 2009;93(1 Suppl 1):3-70.

14. Rabelo E, Aliti G, Domingues FB, Ruschel KB, Brun AO. What to teach to patients with heart failure and why: the role of nurses in heart failure clinics. Rev Lat Am Enfermagem. 2007; 15(1): 165-70.

15. Holland R, Battersby J, Harvey I, Lenaghan E, Smith J, Hay L. Systematic Review of Multidisciplinary Interventions in Heart Failure. Heart. 2005; 91(7):899-906.

16. Michalsen A, Konig G, Thimme W. Preventable causative factor leading to hospital admission with decompensated heart failure. Heart. 1998; 80(5):437-41.

17. Wal MHL, Jaarsma T, Moser DK, Veeger NJGM, Gilst WH, Veldhuise DJ. Compliance in heart failure patients: the importance of knowledge and beliefs. Eur Heart J. 2006; 27(4):434-40.

18. Souza-Rabbo MP, Campos L, Barbosa SR, Rodrigues FSS, Testa RF, Dias MMA, et al. 0 papel de uma equipe multidisciplinar em programas de reabilitação cardiovascular. Ciência em movimento. 2010; 23 (1): 99-106.

19. Mussi CM, Ruschel K, Souza EN, Lopes ANM, Trojahn MM, Paraboni CC, et al. Home visit improves knowledge, self-care and adhesion in heart failure: randomized Clinical Trial HELEN-I. Rev Lat Am Enfermagem. 2013; 21(spec):20-8.

20. Rodríguez-Gázquez Mde L, Arredondo-Holguín E, Herrera-Cortés R. Effectiveness of an educational program in nursing in the self-care of patients with heart failure: randomized controlled trial. Rev Lat Am Enfermagem. 2012;20(2):296-306.

21. Lima MGR, Nietsche EA, BotegaJC, Motta CA, Nicola GDO, Terra LG, et al. Ações educativas na práxis do cuidado em doenças cardiovasculares: um relato de experiência. Rev Enferm UFSM. [Internet] 2012 mai/ago [acesso em 2013 nov 29]; 2(2):449-55. Disponível em: http://cascavel.ufsm.br/revistas/ojs-

2.2.2/index.php/reufsm/article/view/2944

Data de recebimento: $21 / 11 / 2013$

Data de aceite: $24 / 11 / 2014$

Contato com autor responsável: Eneida Rejane Rabelo-Silva

Endereço postal: Universidade Federal do Rio do Sul. Rua São Manoel, 963, Bairro Rio Branco. CEP: 90620-910. Porto Alegre, RS, Brasil

E-mail: eneidarabelo@gmail.com 